\title{
Different mechanisms are responsible for dishabituation of electrophysiological auditory responses to a change in acoustic identity than to a change in stimulus location
}

\author{
Tom V. Smulders ${ }^{\mathrm{a}, \mathrm{b}, *}$, Erich D. Jarvis ${ }^{\mathrm{a}}$ \\ a Department of Neurobiology, Howard Hughes Medical Institute, Duke University Medical Center, Durham, NC, USA \\ ${ }^{\mathrm{b}}$ Institute of Neuroscience, Newcastle University, Newcastle upon Tyne, UK
}

\section{A R T I C L E I N F O}

\section{Article history:}

Received 18 March 2013

Revised 9 July 2013

Accepted 22 August 2013

Available online 30 August 2013

\section{Keywords:}

Zebra finch

Song habituation

Dishabituation

Spatial location

Surprise

Attentional modulation

\begin{abstract}
A B S T R A C T
Repeated exposure to an auditory stimulus leads to habituation of the electrophysiological and immediate-early-gene (IEG) expression response in the auditory system. A novel auditory stimulus reinstates this response in a form of dishabituation. This has been interpreted as the start of new memory formation for this novel stimulus. Changes in the location of an otherwise identical auditory stimulus can also dishabituate the IEG expression response. This has been interpreted as an integration of stimulus identity and stimulus location into a single auditory object, encoded in the firing patterns of the auditory system. In this study, we further tested this hypothesis. Using chronic multi-electrode arrays to record multi-unit activity from the auditory system of awake and behaving zebra finches, we found that habituation occurs to repeated exposure to the same song and dishabituation with a novel song, similar to that described in head-fixed, restrained animals. A large proportion of recording sites also showed dishabituation when the same auditory stimulus was moved to a novel location. However, when the song was randomly moved among 8 interleaved locations, habituation occurred independently of the continuous changes in location. In contrast, when 8 different auditory stimuli were interleaved all from the same location, a separate habituation occurred to each stimulus. This result suggests that neuronal memories of the acoustic identity and spatial location are different, and that allocentric location of a stimulus is not encoded as part of the memory for an auditory object, while its acoustic properties are. We speculate that, instead, the dishabituation that occurs with a change from a stable location of a sound is due to the unexpectedness of the location change, and might be due to different underlying mechanisms than the dishabituation and separate habituations to different acoustic stimuli.
\end{abstract}

(c) 2013 Elsevier Inc. All rights reserved.

\section{Introduction}

The telencephalic auditory system of birds consists of interconnected brain regions with functions and connectivity similar to different layers of the mammalian auditory cortex (Wang, Brzozowska-Prechtl, \& Karten, 2010). These regions consist of Field L2 and the adjacent higher order neurons of L1, L3, the caudal medial nidopallium (NCM) and the caudal mesopallium (CM). L2 is the primary thalamo-recipient neural population similar to the layer IV neurons of mammalian auditory cortex, L1 and L3 are considered similar to layer III neurons of auditory cortex, NCM to layer III of secondary auditory areas, and CM more similar to layers I/II (Fig. 1A; Horita, Wada, Rivas, Hara, \& Jarvis, 2010; Jarvis, 2004; Jarvis et al.,

\footnotetext{
* Corresponding author. Address: Institute of Neuroscience, Newcastle University, Henry Wellcome Building, Framlington Place, Newcastle upon Tyne NE2 4HH, UK. Fax: +44 1912225227.

E-mail address: tom.smulders@ncl.ac.uk (T.V. Smulders).
}

2005; Wang et al., 2010). The caudal striatum (CSt) is also part of the auditory system and is considered similar to the mammalian caudal auditory striatum (Jarvis, 2004). The auditory electrophysiological and EGR-1 immediate early gene (IEG) expression responses in both NCM and CM habituate with repeated presentations of the same song, and exposure to a novel song reinstates the response (Chew, Mello, Nottebohm, Jarvis, \& Vicario, 1995; Mello, Nottebohm, \& Clayton, 1995; Stripling, Volman, \& Clayton, 1997). Long-term retention of the neural habituation is blocked by protein synthesis inhibitors during the initial habituation phase, with the same time course for the dependence of protein synthesis for long term behavioral memories (Chew et al., 1995). Thus, this habituation has been interpreted as the auditory system processing and memorizing the new song, leading to a reduced response as the memory trace is laid down (Stripling et al., 1997). Lu and Vicario (2011) further interpret this encoding of a new neuronal memory as part of the mechanism for identifying auditory objects, with habituation to existing objects facilitating the detection of novel 

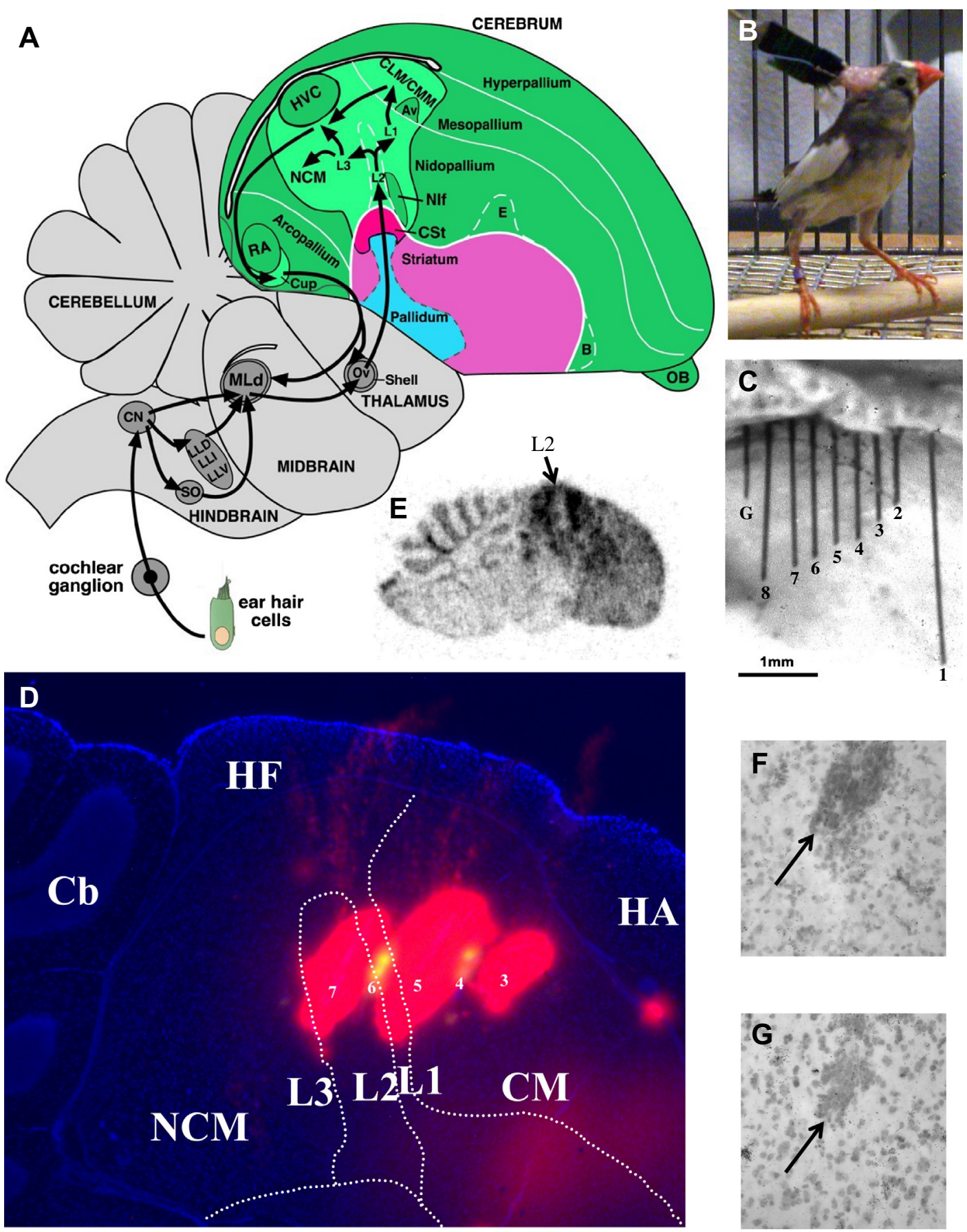

Fig. 1. Auditory pathway and electrode placements. (A) Schematic of auditory pathway, lateral part, in the songbird brain (Jarvis et al., 2005). Only the most prominent and/or most studied connections are indicated. Most of the hindbrain connectivity is extrapolated from non-songbird species. Not indicated are reciprocal connections in the pallial auditory areas. Shown for landmark purposes are song nuclei HVC, RA and NIf. (B) Bird 5 during an experiment. The headstage is visible to the top left. The elastic suspension of the cable makes it light enough for the bird to hold its head normally. (C) Electrode array from Bird 2, inside the skull after dissection. The electrodes are numbered from rostral to caudal ( $G$ = ground electrode). (D) DiI and DiO labeled electrode tracts in a sagittal section (same orientation as in A) through the auditory forebrain of Bird 6. The fluorescently labeled tracts of five electrodes (7, 6, 5, 4, and 3; alternating red and green) aimed at pallial auditory areas are visible in this section, as well as the edges of the tract of electrode 1, aimed at the auditory striatum CSt. DiI (red) spreads more and is more visible than the DiO (green) signal. The dyes leave a trace along the tract of the electrode as the electrode array is lowered into the brain during surgery. Electrode position is marked down the midline of the fluorescent signal, where the track can be seen. The outlines of the anatomical regions are based on adjacent sections, which were stained with cresyl violet and hybridized with a radio-active probe against egr-1 mRNA (see text). (E) An example autoradiogram of an in situ hybridization for the mRNA of egr-1 in the forebrain of Bird 5. Note the strong signal in the auditory pallium, bisected by the light diagonal line of Field L2 (arrow), which has low expression of egr-1. (F and G) Examples of tips of cresyl violet-stained gliosis scars in the forebrain of Bird 5 (electrode 6 (L2; F) and 8 (NCM; G)). Abbreviations: $\mathrm{Cb}$ - cerebellum; CM - caudal mesopallium; CSt - caudal striatum; HA - hyperpallium apicale; HF - hippocampal formation; NCM caudal medial nidopallium.

auditory objects when they occur. The reinstatement of the response to a novel song is then thought to represent the start of the formation of a new memory for a novel auditory object (Lu \& Vicario, 2011; Moorman, Mello, \& Bolhuis, 2011).
Non-acoustic stimuli paired with a song can cause changes in the auditory response to this song. Pairing song with shock under classical conditioning conditions results in less habituation of the IEG response (Jarvis, Mello, \& Nottebohm 1995). Pairing a 
habituated song with a light or playback of a habituated song from a different location leads to dishabituation of the EGR-1 response (Kruse, Stripling, \& Clayton, 2004). Two possible interpretations have been suggested for these findings: (1) The auditory system processes and stores integrated memories that include the tactile, spatial and/or visual context of the sounds, and dishabituation represents the formation of a new such integrated memory of an auditory object (Lu \& Vicario, 2011); or (2) The dishabituation of the auditory response represents modulation by arousal-induced systems (Jarvis et al., 1995; Kruse et al., 2004; Mello, Pinaud, \& Ribeiro, 1998; Ribeiro \& Mello, 2000; Stripling et al., 1997). This latter explanation may be akin to the concept of "surprise", as suggested by Gill, Woolley, Fremouw, and Theunissen (2008).

In the current study, we aim to test in real time whether the auditory system forms integrated neuronal memories of an acoustic stimulus' identity and its spatial location. We performed the experiments on awake, behaving animals, using multi-electrode arrays to record multi-unit electrophysiological activity from several sites in the auditory forebrain simultaneously. We manipulated acoustic identity and location of the songs under various stimulation conditions and found that, like the IEG response (Kruse et al., 2004; Park \& Clayton, 2002), the electrophysiological response in the auditory pathway dishabituates to changes in both acoustic identity and location. However, when stimuli were continuously inter-mixed, habituation was specific to the acoustic stimulus identity, but independent of the changes in location. These findings suggest that the auditory system's response to changes in acoustic identity and in acoustic location is modulated by different mechanisms.

\section{Materials and methods}

\subsection{Animals}

A preliminary description of our approach is described in Jarvis et al. (2002) and Smith, Yu, Smulders, Hartemink, and Jarvis (2006). Six adult female zebra finches (Taeniopygia guttata) from our colony at Duke University were used. Females were chosen for practical reasons, as males were in higher demand for other projects that involved song production behavior and we do not have reason to believe that the auditory system of males and females should behave differently with respect to stimulus acoustic identity and location. Before starting the study, birds were housed in group cages and provided with ad libitum water and standard zebra finch seed mix. All animal procedures were approved by the Duke University Institutional Animal Care and Use Committee.

\subsection{Electrode arrays and implantation surgery}

We chose a stable chronic recording electrode array to obtain multi-unit recordings, instead of a microdrivable one to obtain single units, as we considered anatomical stability across days to be more important. This way we were able to study the response properties of the same small populations of neurons (near the electrode tips) to a wide range of stimuli, in awake behaving birds (Fig. 1B). This was important to us, because we wanted to characterize the response properties of the recording locations in as many ways as possible before finally (after a few weeks) quantifying IEG expression in those same recording locations. The results from the IEG analysis will be reported elsewhere. The current analysis is specifically concerned with the habituation and dishabituation properties of our recording sites, and is based on a subset of recording sessions.

We built multi-electrode arrays by attaching 8 blunt-cut isonel-insulated tungsten microwire electrodes ( $45 \mu \mathrm{m}$ diameter; impedance approximately $0.8 \mathrm{M} \Omega$, range: 0.6-1.2; California Fine Wire) to a nine-channel nano-connector (Omnetics Corp., Minneapolis, MN), using silver paint (SPI Supplies, West Chester, PA). The electrodes were positioned in as straight a line as possible. The rostral-most electrode (electrode 1 ) was situated $\sim 375 \mu \mathrm{m}$ in front of the next electrode (electrode 2), followed by others each spaced $\sim 250 \mu \mathrm{m}$ apart. The electrodes were cut in such a way that electrode 1 was the longest and electrodes $2-8$ were the shortest to the next longest, at an angle of $30^{\circ}$ from the horizontal (Fig. 1C). This configuration ensured that during surgery, electrode 1 was aimed at the auditory caudal striatum (CSt), while the other seven covered the stretch from auditory CM through L1, L2, L3, to NCM (Fig. 1A and D). A ninth electrode was added either behind electrode 8 or in front of electrode 1 as a ground (with a large section of insulation removed; in birds 1-4) or as a reference electrode similar to the other electrodes, but implanted in a non-auditory brain region anterior to $\mathrm{CM}$ (in birds 5 and 6). In the latter case, a separate silver wire was used as the ground electrode. This setup (with separate reference and ground electrodes) significantly reduced the amount of movement artifact.

Before implant, fluorescent Dil (red) and DiO (green) dyes (Molecular Probes, Eugene, OR) were applied and dried to alternating electrode tips in the array, such that their locations in the brain could later be identified. The dyes did not interfere with the electrode recordings nor with the types of responses the neurons gave when compared to a control implant that had electrodes without dyes (see also DiCarlo, Lane, Hsiao, \& Johnson, 1996; Naselaris, Merchant, Amirikian, \& Georgopoulos, 2005). The arrays were implanted under isoflurane anesthesia. Birds were fixed in a custommade stereotaxic frame (H. Adams, CalTech) with the head held at a 45 degree angle. A rectangular opening was made in the skull above the auditory areas of the right hemisphere and the array was lined up parallel to the midline of the brain. Electrode 1 was zeroed to the split in the mid-sagittal sinus (visible through the bottom layer of the skull), maneuvered $2.75 \mathrm{~mm}$ rostral, $0.45-$ $0.7 \mathrm{~mm}$ lateral (slightly different coordinates in different birds), and then lowered to $\sim 2.7 \mathrm{~mm}$ below the surface, with the other electrodes following in their pre-configured positions. Every time an electrode almost touched the dura mater, a small slit was cut into the dura to allow the electrode to slip in. As the electrode array was advanced, electrophysiological activity on each electrode was recorded using an extracellular amplifier (FHC Inc., Bowdoinham, ME) and custom-written software in LabView $\odot 6.1$ (National Instruments, Austin, TX). The LabView software was based upon that developed in the laboratory of Richard Mooney (version of Fred Livingston and Merri Rosen (Rosen \& Mooney, 2000; Rosen \& Mooney, 2003)). To search for auditory responses, the experimenter whistled scales. When at a depth where all or most of the electrodes showed increased multi-unit responses to the whistles, the array was left in place and attached to the skull using dental cement and cyanoacrylate glue. The birds were allowed to wake up and were eating and drinking within $20 \mathrm{~min}$. After $\sim 2$ days of recovery, they were attached to a commutator recording set-up (described below) in a soundproofed anechoic room.

\subsection{Electrophysiological recording and signal processing}

Throughout the experiment, the birds were connected through a lightweight recording cable with headstage (Plexon Inc., Dallas, TX; Fig. 1B) to a motorized commutator (Dragonfly Inc., Ridgeley, WV) at the top of the cage. The birds habituated to this chronic tether overnight, after which the experiments were started. Once habituated, the birds moved around freely in their cage, eating, drinking, hopping, sleeping and grooming. The headstage contained one unit-gain op-amp for each channel, including the reference wire, to match the impedance of the wires to the impedance 
of the amplifier. The reference channel was split into a non-amplified ground channel and an amplified reference channel for birds $1-4$, whereas for birds 5 and 6 the connection between ground and reference was cut to reduce movement artifact. The signals from the commutator were sent to a multi-channel extracellular amplifier (500× amplification, band-pass filtered between $220 \mathrm{~Hz}$ and $5.9 \mathrm{kHz}$; Plexon, Inc., Houston, TX) and from there onto a PCI-6071E Analog to Digital conversion card (National Instruments, Austin, TX) in the computer. The electrophysiological recording was triggered by the start of the playback (i.e. the silent period before the stimulus proper; see below). The sound in the recording room (including the playback stimulus, calls by the bird itself, movement of the commutator motor, etc. if they were present) was recorded using a Sennheiser ME62 microphone, amplified via a Midiman microphone amplifier, fed into the same PCI-6071E card. Sound and the multi-unit electrophysiological signals were all digitized in parallel at $20 \mathrm{kHz} /$ channel using our custom-written LabView software.

Since in birds 1-4 the ground and reference channels were connected to the same wire, when the bird moved around, there were often strong movement artifacts, which were identical on all channels. To remove this artifact, we incorporated an Independent Component Analysis (ICA) Matlab routine (Makeig, Bell, Jung, \& Sejnowski, 1996; Makeig \& et al., 2000) into our custom-written LabView software. This routine searched for a component that contributed approximately equally to all 8 recording channels in any given playback trial. If this component was present, it was then deleted and the information on the channels reconstituted using the remaining 7 components (see Supplementary materials; Fig. S1). This approach is commonly used in EEG recordings to remove movement or other artifacts that occur across many channels (Barbati, Porcaro, Zappasodi, Rossini, \& Tecchio, 2004; Brown, Yamada, \& Sejnowski, 2001; Delorme \& Makeig, 2004; Iriarte et al., 2003; Joyce, Gorodnitsky, \& Kutas, 2004; Jung et al., 2000; Tran, Craig, Boord, \& Craig, 2004). Depending on the bird's behavior, such a component was detected and subtracted on 14-94\% (median $38 \%$ ) of trials in any given recording session (i.e. across all conditions in a given session). Removing this component from all 8 channels usually resulted in a lower RMS power across all channels for the trial in question (compared to trials during which no movement artifact had been removed). To statistically control for this difference in RMS power among trials within one experiment, we coded for all trials whether the ICA algorithm had been applied as a dummy variable in any subsequent analyses. The ICA was not applied to birds 5 and 6 , since the differential recording setup had mostly eliminated movement artifact of this type.

\subsection{Playback conditions}

For the entire duration of the experiment (approximately 3 weeks), a bird was housed in the soundproofed, anechoic recording room in a $43 \times 60 \mathrm{~cm}$ cage, while being tethered to a recording cable, which allowed free movement throughout the cage (see above; Figs. 1B and 3A). Around the cage were four speakers (Cambridge Soundworks Creative CSW1500 surround sound gaming speakers connected to a SoundBlaster Live card) placed roughly $50 \mathrm{~cm}$ from each corner of the cage, from which stimuli were played back. The recording room was separated from an anteroom, in which the experimenter was located together with all the recording equipment, by a one-way window. Through this window and a TV monitor in the recording room connected to a SONY DCRPC100 Digital HandyCam, the bird's behavior was monitored. The camera was also used to record the behavior to mini-DV tapes when required. The birds had ad lib food and water in the cage and the lights were set to a 12/12 L/D schedule. All exposure was passive, by which we mean that a bird was allowed to do what it wanted to do during the playback.

Our stimuli were WAV files, which we manipulated so they would all generate the same average power (measured as the Root Mean Square (RMS) of the signal). We did this by playing them in a soundproof box using the same speakers as used in the playback experiment, re-recording them (digitized at $20 \mathrm{kHz}$ ) using a microphone with a flat spectral response profile (Radioshack lapel microphone), and then measuring the RMS of the microphone-recorded signal. Stimuli were then adjusted until they all produced a similar RMS and hence average power. In the anechoic recording room, the speaker level was set so that a $1 \mathrm{kHz}$ pure tone played from two speakers on the long side of the rectangle cage simultaneously delivered an $80 \mathrm{~dB}$ SPL in the center of the cage (Radioshack $\mathrm{dB}$ meter). All songs used were recorded from birds in Dr. Richard Mooney's aviary at Duke University, and were therefore from males unfamiliar to the females from Dr. Jarvis' aviary. Each song stimulus consisted of two motifs, without introductory notes. All stimuli were preceded and followed by a period of silence of exactly the same length as the stimulus itself, resulting in a total length of between 3.6 and $8.6 \mathrm{~s}$ (silence-stimulus-silence), depending on the length of the stimulus.

The playback conditions described below are a subset of a larger number of playback sessions performed on each of the birds over the first five days of the experiment. Of these sessions, we chose 4 that were run on Days 2 and 3 of the experiment for the current analysis, such that the recording circumstances would be as similar as possible to each other.

\subsubsection{HabAcoustic: repeated playback of one song, followed by a novel song}

We played the same song (S1) 50 consecutive times simultaneously from the two speakers along one of the long sides of the rectangle, with a start-to-start interval of $15 \mathrm{~s}$, followed by 50 consecutive iterations of a song from a second bird (S2), then 50 iterations of a third bird's song (S3) and finally another 50 from a fourth bird (S4), for a total of 200 song playbacks. The consecutive playbacks of the song from the same bird were always of the same identical recording. The four different songs were selected such that they were of approximately the same length. All birds experienced the same four songs in the same order.

\subsubsection{HabLocation: repeated playback of one song, followed by the same song from a different location}

We played a novel conspecific song (S5) 50 times from one of the speakers, then switched that same song to the speaker diagonally opposite the original location for another 50 iterations, and then switched it back to the original speaker for a final 50 iterations. In the HabLocation condition, Loc1 was $225^{\circ}$ (except for Bird 1 , for which it was $315^{\circ}$ ) and Loc 2 was $45^{\circ}$ (except for Bird 1 , for which it was $135^{\circ}$; Fig. $3 \mathrm{~A}$ ). The inter-stimulus interval was the post-stimulus silence of the previous stimulus plus the pre-stimulus silence of the second stimulus, which added up to approximately $5.3 \mathrm{~s}$.

\subsubsection{MixAcoustic: 8 different sound stimuli played in a pseudo- random order}

We played 8 stimuli of roughly the same length $(2 \mathrm{~s})$ in pseudorandom order: (1) one two-second WN stimulus (WN); (2) a Bengalese finch song (Beng); (3-4) two different conspecific zebra finch songs (S6 and S7); (5) one of these songs played in reverse (S6R); (6) the same song played with the syllable order reversed (S6RO); (7) the same song with the syllables themselves reversed but played in the correct order (S6RS); and (8) white-noise with the amplitude envelope of this song (S6WN). The 8 different stimuli were played in random order until all 8 had been used. Then all 
8 were played again, now in a different random order until all 8 were used up, and so on for a total of 20 times. The length of the inter-stimulus interval was the duration of the post-stimulus and pre-stimulus silence, which was approximately $4 \mathrm{~s}$. All birds experienced the same 8 stimuli. However, the order was randomized independently for each set of 8 play-backs, so different birds experienced the stimuli in a different order.

\subsubsection{MixLocation: the same stimulus played from 8 different locations in a pseudo-random order}

We presented the same song (S8) pseudo-randomly from 8 different locations around the cage. The locations corresponded to the 4 individual speakers and to 4 locations exactly in between each pair of speakers (by playing from pairs of adjacent speakers; Fig. 3A). The song was played from these 8 locations in a random order until all 8 had been used. Then we again played the song from all 8 in random order until all 8 were used, and so on for a total of 20 times, as in the MixAcoustic condition. No extra time was added in addition to the silence before and after the sound that was part of the wav files, resulting in inter-stimulus intervals of approximately $5.7 \mathrm{~s}$. All birds experienced the same song in the MixLocation condition, but the order of the locations was unique to each bird, as in the MixAcoustic condition.

During the HabLocation and MixLocation conditions, the birds' head directions were video recorded (except Bird 1, for which we did not have video recordings) and later analyzed to score the bird's initial and final head directions relative to the speakers for each stimulus presentation and sorted into two categories: sound coming from the left and sounds coming from the right of the bird's head. Initial and final head directions correlated very tightly (circular correlation coefficient for HabLocation condition: 0.411-0.712, all $p<0.001$; (Fisher, 1993), and we therefore only used initial head direction in the subsequent analyses.

\subsection{Anatomical localization of the electrodes}

After having undergone these and a range of other stimulation and recording sessions for other studies (Jarvis et al., 2002; Smith et al., 2006), the birds were killed at the end of the 3rd week of the experiment. In the last recording session, the birds were exposed to 50 iterations of a novel conspecific song (S9) for $15 \mathrm{~min}$ to induce IEG gene expression in the auditory system. We then waited another $10 \mathrm{~min}$ to let the mRNA accumulate before quickly decapitating the birds, dissecting out the brain and fast-freezing it. Brains were stored at $-80^{\circ} \mathrm{C}$ until processing. Brains were cut on a cryostat into four or six alternate series of $10 \mu \mathrm{m}$ sagittal sections. One series was stained with DAPI and coverslipped for examination of the alternating fluorescent Dil and DiO electrode tracts (Fig. 1D). Another series was hybridized with a radioactive RNA probe for egr-1 using a previously established procedure (Jarvis \& Nottebohm, 1997) to visualize known gene expression differences in the auditory forebrain areas (Mello \& Clayton, 1994) (Fig. 1E). Thus, we had four sources of information to anatomically identify the electrode locations: (1) we used the gliosis along the electrode tracts to locate the tips of the electrode tracts in cresyl-violetstained tissue sections (Fig. 1F and G); (2) we then used the fluorescent dye from the electrode tips to identify each electrode (Fig. 1D); finally, we used (3) hearing-induced EGR-1 gene expression patterns (with L2 showing much lower EGR-1 levels relative to the surrounding nidopallium (Fig. 1E)) and (4) known cresylviolet defined boundaries among brain regions, to localize the electrode tips to a particular brain region.

All electrode tracts were found in all birds and assigned to one of six auditory forebrain areas (Fig. 2): NCM, Field L3, Field L2, Field L1, CM, and CSt. CM has topographic medial (CMM) and lateral (CLM) subdivisions that are interconnected respectively with

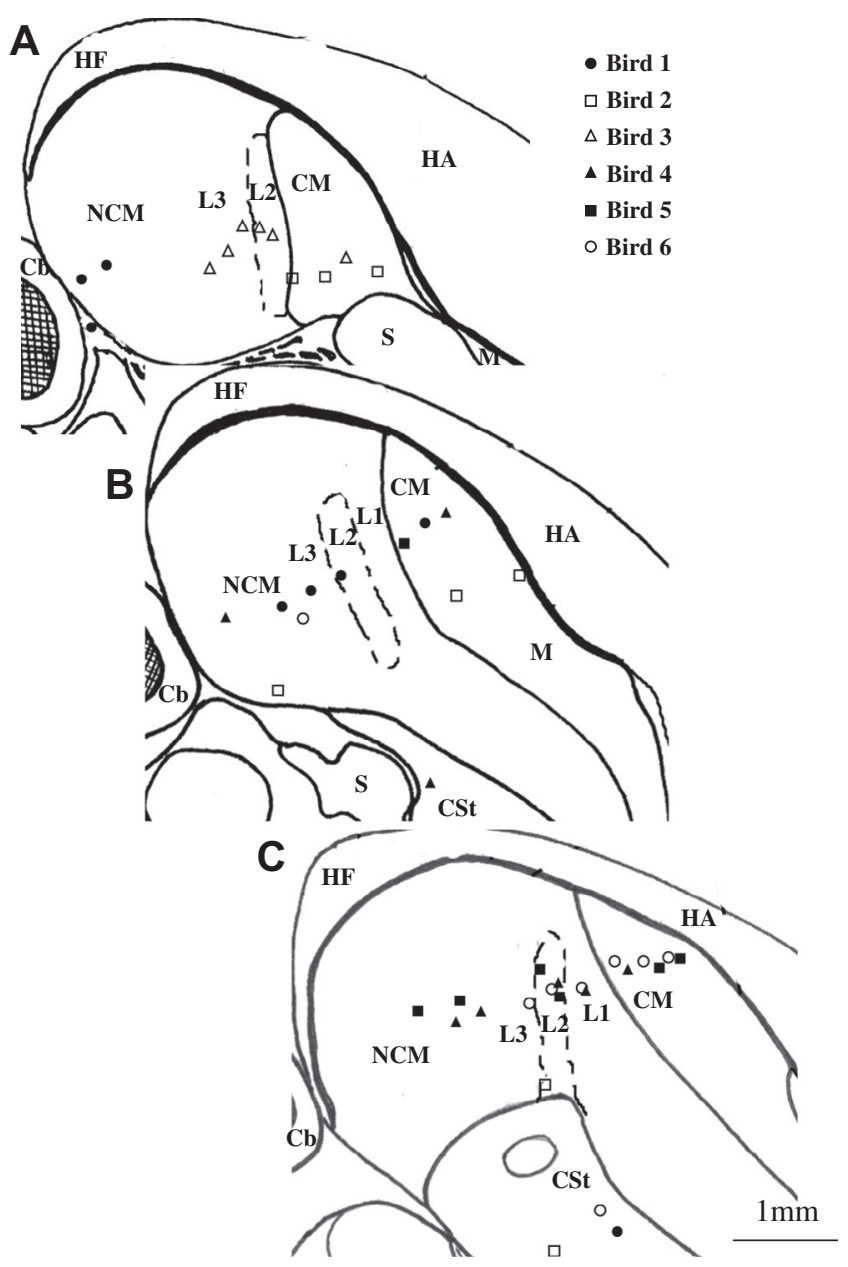

Fig. 2. Approximate locations of all electrode locations from which auditory responses were recorded. Drawings represent sagittal sections at (A) $\sim 0.4 \mu \mathrm{m}$, (B) $\sim 0.6 \mu \mathrm{m}$; and $(\mathrm{C}) \sim 0.8 \mu \mathrm{m}$ from the midline. Electrode locations from different birds are indicated with different symbols. The rostral end of the brain is to the right, and dorsal part is at the top of the figure. Abbreviations are as in Fig. 1, except men - meninges; $\mathrm{S}$ - septum.

medial and lateral parts of the auditory nidopallium (Vates, Broome, Mello, \& Nottebohm, 1996); our definition of CM includes both medial and lateral parts. To assign the electrodes to these specific brain areas, we looked for the section that contained the electrode tip (deepest point of the tract) and determined its location in the auditory forebrain relative to the different laminae. Since the boundary between L3 and NCM is not easy to determine in Nissl stain or with EGR-1, we estimated it based on the distance from L2 ( $\sim 0.4 \mathrm{~mm})$. Because we only had small numbers of recording sites in each brain region, to generate quantitatively more accurate anatomical comparisons across birds we coded our recording sites based on their position relative to L2 (the thalamorecipient region), with sites in L2 being ranked " 0 ", the first non-L2 site (both rostral and caudal of L2) ranked " 1 ", the next " 2 ", etc.

\subsection{Data analysis}

\subsubsection{Response strength}

The neuronal density is very high in the auditory system of small songbirds, and our low-impedance electrodes therefore recorded from multiple units simultaneously. There were too many units to even be able to "isolate" multi-unit spikes. Therefore, to quantify the amount of neural activity in the population of neurons near our electrode tips, we used the RMS of the voltage signal as 

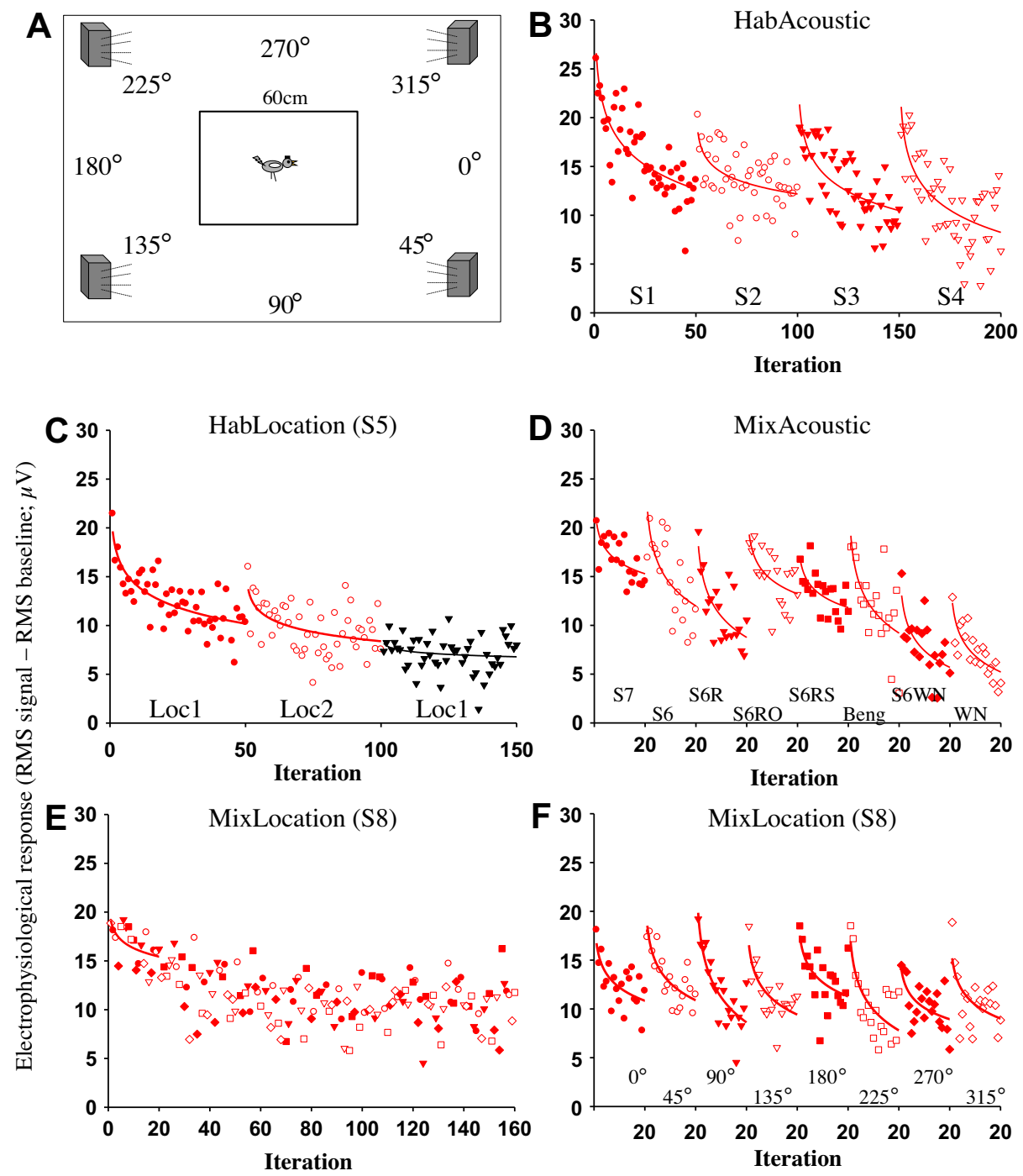

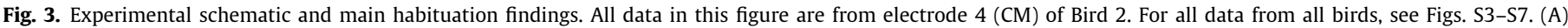

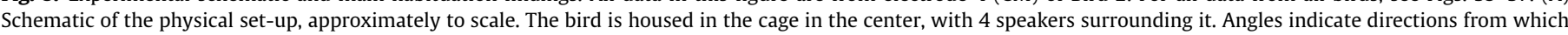

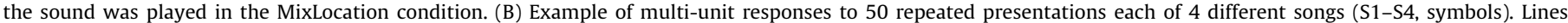

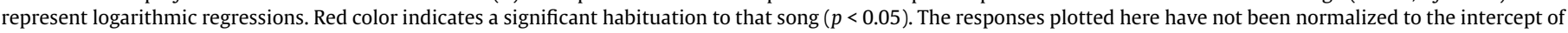

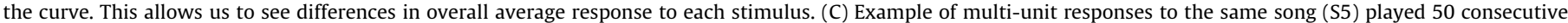

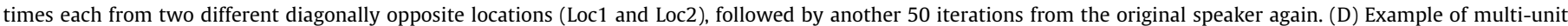

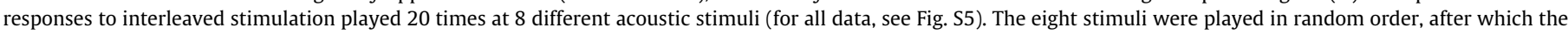

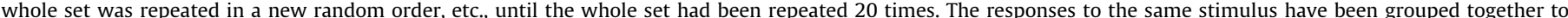

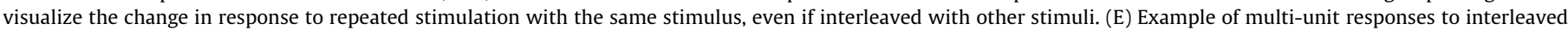

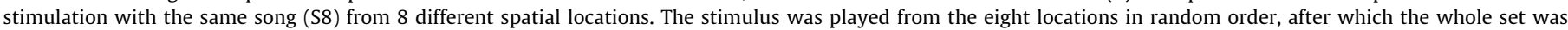

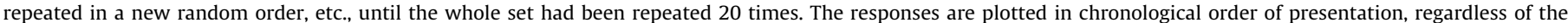

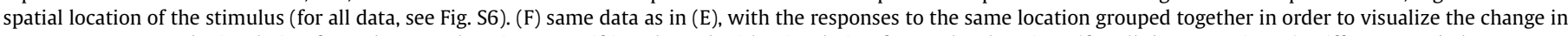

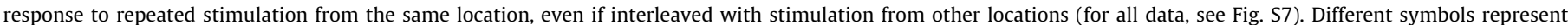
different stimuli or different locations, depending on the condition.

our response measure. This is consistent with previous work in this system (e.g. (Chew et al., 1995). We calculated the RMS of the electrophysiological signal during the silent period before the onset of the stimulus (i.e. baseline activity), and again for the period of the stimulus itself. The raw response strength to this particular stimulus was then calculated as the difference between the RMS during the stimulus and the RMS of the baseline activity (Fig. S2). These values were calculated using our custom-written LabView software and exported to SAS 9.2 for Windows (SAS Institute, Cary, $\mathrm{NC}$ ) for further processing and analyses.

\subsubsection{Controlling for confounding variables}

Because we are mainly interested in the habituation slope of the responses to repeated exposure to the same stimulus, we wanted to get as good an estimate of this slope as possible. We therefore controlled statistically for the two other variables that we found had a significant effect on the size of the response in a given recording site: (1) removal of movement artifact by ICA and (2) head direction. To control for the removal of movement artifacts by ICA (for birds $1-4$, as outlined earlier), we simply used a dummy variable to identify those trials in which artifact had been removed to statistically control for the effect of this process on the signal strength. To control for head direction in the HabLocation and MixLocation conditions (in which the sound came from only one speaker and was therefore much more directional than in the HabAcoustic and MixAcoustic conditions), we used a dummy variable to indicate whether the sound came from the left or the right side of the head. Then we ran a regression with the relevant 
confounding variables as the independent variables and the response strength as the dependent variable. We collected the residuals from this regression for further analysis. Because residuals have a mean of zero, we added the mean predicted value from the regression across the entire recording session to each residual, which resulted in values that were similar to the original response strength values, but with the variation due to confounding variables subtracted.

\subsubsection{Measuring habituation rates}

Habituation does not occur linearly, as the drop in response is steeper in the first few iterations than in the later ones (Chew et al., 1995; this study). We therefore fit logarithmic functions to the data, by regressing adjusted response strength (see above) against the natural logarithm of the iteration (i.e. response $=a^{*} \ln ($ iteration $)+b$ ). We identified outliers, if any, which we defined as data points whose studentized residuals from the logarithmic regression were larger than 3 . These outliers were then excluded from the analysis and the regression run again without them. To quantitatively compare the rate of habituation among different conditions, we normalized the responses in each regression to the intercept of that regression by dividing each response by the intercept, multiplied by 100 . We then re-ran the identical regression as before on the normalized responses. The significance of these regression analyses is identical to the original outcomes, but the intercept is now equal to 100 , and the slope is now comparable across conditions (Chew et al., 1995). Sites were considered to habituate to a given repetition of stimuli if the logarithmic regression on the normalized responses resulted in a significant negative slope.

\subsubsection{Comparing habituation rates across stimuli and conditions}

In order to compare slopes of two regression lines, we ran a General Linear Model (GLM) analysis with the normalized responses from the two conditions of interest as the dependent variable, the natural logarithm of the iteration as a co-variate and a variable identifying the condition as a between-subjects factor. For each recording site, we then looked for a significant interaction between condition and iteration as evidence for a difference in habituation rates. If the interaction was not significant, the habituation rates were considered not significantly different from each other.

\subsubsection{Comparing response strength across stimuli}

In order to compare the strength of the response to two different stimuli, we ran the same GLM analysis as outlined above, but this time with the non-normalized responses from the two stimuli of interest as the dependent variable. For each recording site, we then looked for a significant main effect of the stimulus, while controlling for the fact that the response habituates over time.

\subsubsection{Defining dishabituation}

When, after habituation to one stimulus, a new stimulus is presented (or the old one under different circumstances), the response to the new stimulus is sometimes greater again than the habituated response. We interpret this increase in response as a form of dishabituation (although this term strictly speaking only applies if the stimulus is the same), analogous to the behavioral Habituation-Dishabituation Paradigm often used with infants and non-human animals (Bornstein, 1985), and use it to discover which changes to a stimulus are salient to the system under investigation. In our experience, the absolute magnitude of the response after the change in stimulus is not the best measure of dishabituation, as a particular brain area may respond more strongly to one stimulus than another, regardless of habituation. This is especially true in the HabAcoustic and the MixAcoustic conditions, where the stimuli are acoustically different from each other. To avoid this potential confound we used rate of habituation as a measure of dishabituation. We considered a brain area to dishabituate after exposure to a habituated stimulus if it habituates to the novel stimulus at the same rate as it did to the original stimulus. If the change in the stimulus was not salient to the system under investigation, then the habituation should continue as if nothing had changed, resulting in a much shallower habituation slope (potentially not different from zero) to the new than to the original stimulus (Chew et al., 1995). Our operational definition of dishabituation was therefore the following: if the response to the new stimulus significantly habituated (criteria above) AND if the slope of the logarithmic regression for the second stimulus was not significantly shallower than the slope of the first stimulus. Slopes were compared as explained above.

\subsubsection{Generalizing results across recording sites and across birds}

We performed statistical tests for each recording site to determine whether it habituates, what the rate of habituation is, and whether it differs from the rate of habituation under a different condition. In order to combine the different statistical tests across all the recording sites and birds, we applied a technique from meta-analysis called Stouffer's pooled $z$-test (Whitlock, 2005). For this test, one first needs to define a one-tailed prediction about the difference under investigation (e.g. "slopes should be shallower in condition $\mathrm{X}$ than in condition Y"). In some cases, this one-tailed prediction was based on the hypothesis we were testing. For example, when testing whether dishabituation takes place with a certain manipulation, the question is whether the slope is shallower after the change than before the change. If so, the sites do not dishabituate. In many cases, however, there was no a priori reason to pick one one-tailed prediction over another. In those cases, we always chose the prediction that was consistent with the direction of the average difference as present in the data, as we wanted to know whether this difference was significant or not. In all cases in which we found significant results, the $p$-values were smaller than 0.025 , and therefore would have been significant if the prediction had been two-tailed as well. According to Stouffer's method, we calculated the one-tailed $p$-values for that prediction and converted these $p$-values into a $z$-score. These $z$-scores were then combined by adding them up and dividing this total by the square root of the number of tests being combined (e.g. when combining the results of 8 recording sites, the sum of $z$ scores is divided by the square root of 8 ). Because the sites that were recorded from the same bird were not independent of each other (they were recorded simultaneously), we applied Stouffer's pooled $z$-test in a 2-stage process: first we combined all the sites within each bird; and second we combined the $z$-scores for each bird across all birds in the same way. This allowed us to look for general properties that apply across recording sites and birds, taking into account that sites are nested within birds. When we looked at differences in habituation slopes between two conditions, we only included those sites in the analysis that habituated in both conditions under comparison. This is because the concept of a habituation slope really only applies in a site that habituates in the first place. We also used Stouffer's test to combine results from different comparisons to draw a more general conclusion.

All other statistics used were routine, and included $\chi^{2}$ contingency tables for comparing proportions of sites that habituate or dishabituate and Spearman rank correlations for looking at the association between variables. Results were considered significant if $p<0.05$.

\section{Results}

Of the 48 electrodes implanted across 6 animals, 44 sites responded significantly to the playback of conspecific song and were 
in the auditory pathway. These were 4 sites located in the caudal striatum (CSt; across 4 birds), 15 in the caudal mesopallium (CM; across 6 birds), 8 in L2 (across 6 birds), and 17 in the auditory caudal nidopallium (L1, L3, NCM; across 6 birds) (Fig. 2). Of the remaining 4,1 was located outside of neural tissue in the meninges (in Bird 1), 1 in a non-auditory part of the striatum (in Bird 5), and two channels were short-circuited with each other (in Bird 3).

\subsection{Song identity habituation in freely behaving animals}

Previous electrophysiological recordings for habituation studies from the auditory system of zebra finches have been made in either anaesthetized or awake, but restrained birds (e.g. Chew, Vicario, \& Nottebohm, 1996; Chew et al., 1995; Stripling et al., 1997). We therefore tested whether song habituation would occur in awake and freely-behaving birds. In the HabAcoustic condition (i.e. the repetition of the same novel conspecific song (S1) 50 times, followed by 50 iterations of a second (S2), third (S3) and fourth (S4) novel conspecific song respectively; see Section 2.4 ), $72 \%$ of the sites habituated to 50 iterations S1, including several sites in L2 and CSt (Figs. 3B and S3). Upon the introduction of 50 iterations of subsequent songs, $85 \%$ of the sites that had originally habituated to S1 habituated again at the same rate to S2, 77\% to S3, and $92 \%$ to S4 (Figs. 3B and S3). The proportions that dishabituated to these three songs were not different from each other $\left(\chi^{2}(1)<2.36\right.$, $p>0.12$ ). The habituation slopes to the four songs correlated significantly with each other $(r>0.582, p<0.001, n=36)$, showing overall consistency in habituation slope within recording sites. Mean response strengths to songs $2-4$ were consistently lower than to song $1\left(Z_{c}<-6.95, p<0.001\right)$ across all birds, but not consistently different among songs $2-4\left(Z_{c}>-1.07, p>0.14\right)$. These findings demonstrate that neural activity habituation to repeated playback of zebra finch song and the dishabituation to the introduction of a novel song in the auditory system occurs robustly in awake and behaving zebra finches.

\subsection{Auditory responses are sensitive to changes in spatial context}

We next investigated whether a change of spatial location from which the song was presented affected the auditory response strength. First, we found that the response to sounds coming from the side of the head contralateral to the implant was stronger than the response to sounds coming from the side of the head ipsilateral to the implant $\left(Z_{c}=-6.48, p<0.0001\right.$; Fig. 4B). The magnitude of the difference between sounds coming from the contralateral vs. ipsilateral side of the head in units of standard deviations (Cohen's d) did not vary with anatomical location (i.e. distance from L2; Spearman's Rho $=-0.055, p=0.759, n=34$ ). Because the birds could move around freely, this meant that sometimes the sound was coming from their ipsilateral, and sometimes from their contralateral side. As outlined in the Materials and Methods, we controlled for this extra source of variance in the auditory response in the habituation analyses for the HabLocation and MixLocation conditions by using a regression and residual approach to normalizing response strength by head direction.

In the HabLocation condition (i.e. 50 repetitions of S5 from one location, followed by 50 repetitions of $\mathrm{S} 5$ from a different location, and finally 50 repetitions of S5 from the original location again; see Section 2.4), we found that, similar to the first song in the HabAcoustic identity condition (S1), 73\% (32/44) of the sites across 6 birds habituated to 50 iterations of the same song (S5), played from one speaker (including 4 sites in L2 and one in CSt). The proportion of sites that showed habituation to S5 was no different than the proportion that habituated to S1 in the HabAcoustic condition $\left(\chi^{2}(1)=0.003, p=0.96\right)$. When the same song (S5) was switched to coming from a different location, $56 \%(18 / 32)$ of the originally habituating sites showed dishabituation (Figs. 3C and S4). This proportion of sites that dishabituated to a change in song location was significantly lower than the proportion that dishabituated to a change in acoustic identity of the song $\left(\chi^{2}(1)=5.39, p=0.02\right)$. When the song was moved back to the original location for another 50 iterations, only $25 \%(8 / 32)$ of the original habituating sites dishabituated, which is significantly fewer sites than to the first change in location $\left(\chi^{2}(1)=6.48, p=0.011\right.$; Fig. S4). We did not find any statistical difference in the distribution of sites that dishabituated in the HabLocation versus HabAcoustic conditions $\left(\chi^{2}(1)=1.83, p=0.18\right)$.

Similar to the HabAcoustic condition, the mean response strengths after the first and second change of location were lower than to the first location $\left(Z_{c}<-7.98, p<0.001\right)$. However, in contrast to the HabAcoustic condition, in the HabLocation condition, after the second location switch, the mean response strength was lower still than after the first switch $\left(Z_{c}=-5.08, p<0.001\right.$; including all sites in all birds; e.g. Fig. $3 C$ ). This indicates that despite more than one change in location, the response to the moving song (S5) continues to habituate further with further exposure.

\subsection{Habituation to intermixed acoustic stimuli is similar to habituation to songs played separately}

To investigate whether the auditory system treats different sound stimuli played from the same spatial location as different auditory objects, we looked at the habituation of the auditory responses to different stimuli that were played back intermixed with each other (the MixAcoustic condition). We presented 20 blocks of playback of 8 different stimuli, including 2 different zebra finch songs (S6 and S7), 4 manipulations of one of these songs (S6R, S6RO, S6RS and S6WN), a Bengalese finch song (Beng) and white noise (WN) intermixed randomly within each block (see Section 2.4). If the auditory system considers them to be separate objects, it should habituate to them independently of the other stimuli with which they are intermixed. If the auditory system considers them to be the same objects, habituation should continue across stimuli.

Before determining the effects on habituation, we first asked if the auditory responses were similar to the different stimuli. We found that in the MixAcoustic condition most sites in the auditory system preferentially responded to zebra finch song compared to heterospecific Bengalese finch song, white noise modulated with a zebra-finch song amplitude envelope, and pure white noise (Fig. 4A: comparing S6 and S7 versus Beng, S6WN, and WN). This is similar to isolated non-mixed stimuli (Chew et al., 1995). However, there were 6 sites across 4 birds that actually responded most strongly to white noise (WN) and white noise modulated with a zebra-finch song amplitude envelope (S6WN; 3 in L2 and 3 in the adjacent auditory nidopallium; Fig. 4A). Responses to S6 were not different from responses to the same song played in reverse, with reversed syllables or with syllables in reverse order (Fig. 4A panels labeled S6R, S6RS and S6RO in the first column). These findings suggest that even in the MixAcoustic condition, the auditory system distinguishes most stimuli as it does when they are played independently.

In terms of habituation, we found that each stimulus had on average $50 \%$ (22/44) of the sites habituating to it (range: $43-56 \%$; Figs. 3D and S5). The proportions for each stimulus were not significantly different from each other $\left(\chi^{2}(1)<1.64, p>0.200\right)$ or from the proportion of sites significantly habituating to the first 20 renditions of S1 in the non-mixed HabAcoustic condition $\left(\chi^{2}(1)<1.71\right.$, $p>0.191)$. This indicates that habituation to intermixed sounds is similar to habituation to sounds played separately. Controlling for the differential responses to different stimuli, we asked whether the habituation slopes of the responses to the 20 itera- 

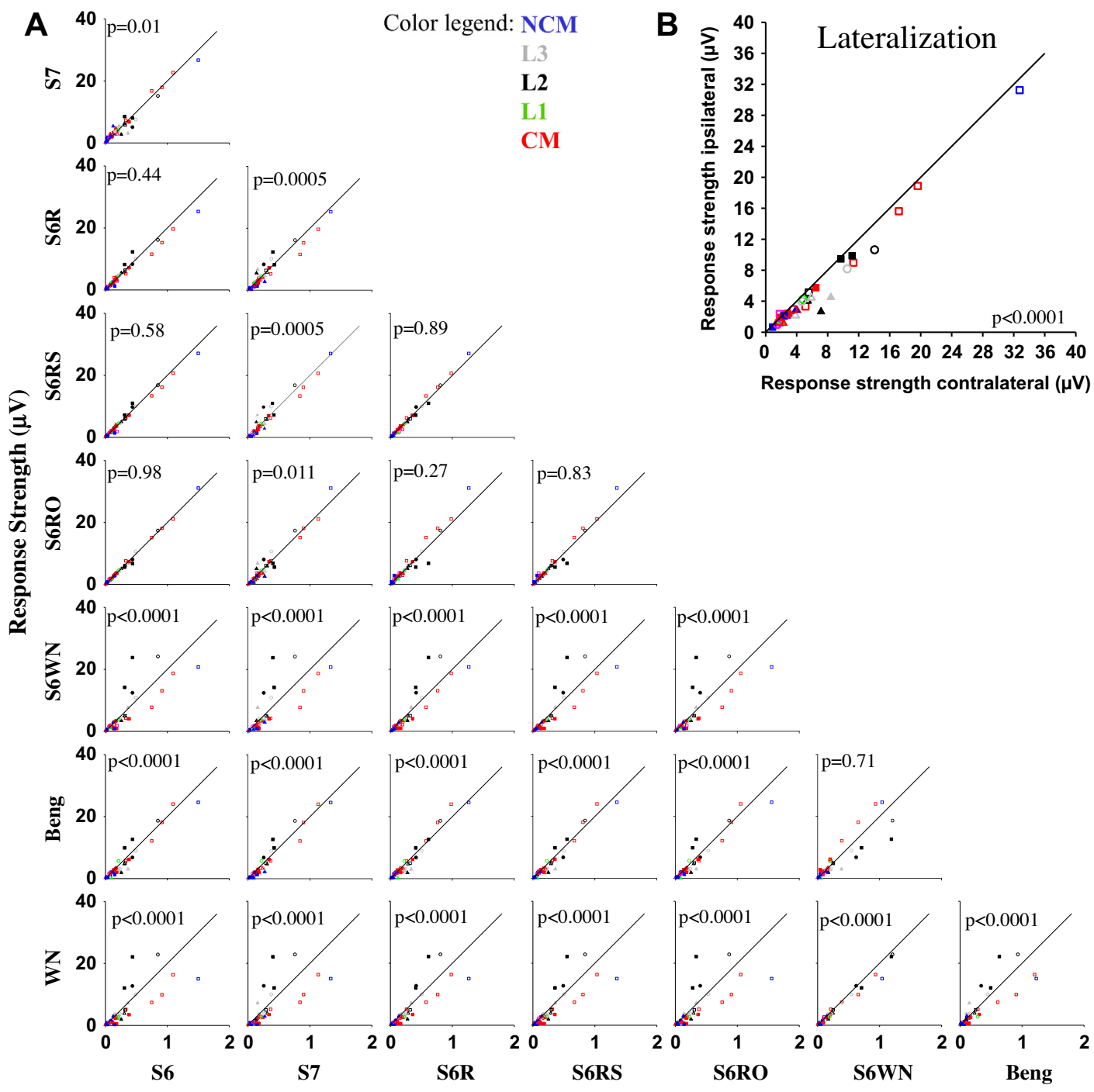

Response Strength $(\mu \mathrm{V})$

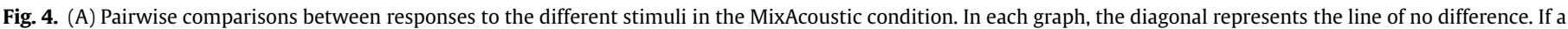

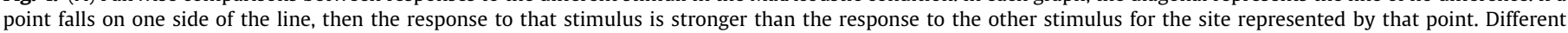

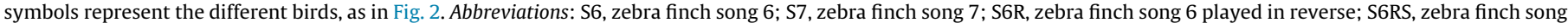

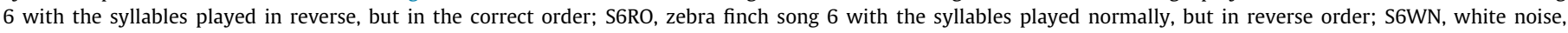

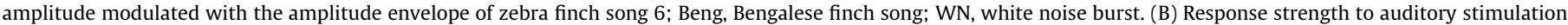

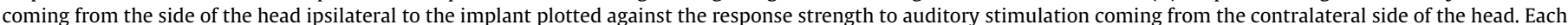

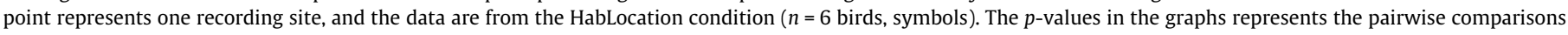

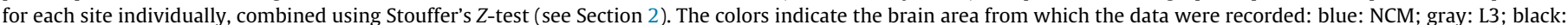
L2; green: L1; red: CM; pink: CSt.

tions each of the two zebra finch songs (S6 and S7) in the MixAcoustic condition were similar to the habituation slopes of the responses to the first 20 iterations of S1 in the HabAcoustic and S5 in the HabLocation conditions. We found that the habituation slopes to S6 and S7 in the MixAcoustic condition did not differ significantly from $S 5$ in the HabLocation condition $\left(Z_{c}=0.89\right.$ to $-0.915, p>0.18$ ), nor did the habituation slope to $S 7$ differ from $S 1$ in the HabAcoustic condition $\left(Z_{c}=-0.437, p=0.331\right)$; however, S6's slope in the MixAcoustic condition was just significantly steeper than S1's in the HabAcoustic condition $\left(Z_{c}=-1.94, p=0.026\right)$. The habituation slopes to $S 6$ and $S 7$ did not differ significantly from each other $\left(Z_{c}=-0.941, p=0.17\right.$; Figs. $5 \mathrm{~A}$ and $\left.\mathrm{S} 8 \mathrm{~A}\right)$.

We investigated why the habituation slope to $S 6$ was slightly steeper in the MixAcoustic condition, and found that the habituation slopes to the different acoustic manipulations of S6 varied, with the steepest slopes for white noise envelope of the song
(S6WN) and the shallowest for Reversed-order syllables (S6RO). Pairwise comparisons showed that the only significant difference was that Reversed Song (S6R) had habituation slopes that were significantly steeper than Reversed Order song (S6RO) $\left(Z_{c}=-1.83\right.$, $p=0.033$ ). If we make the assumption that the habituation slopes to the different versions of S6 are not different from each other, then the question becomes: are they steeper than single songs (S7 from the MixAcoustic condition, S1 from the HabAcoustic condition and S5 from the HabLocation condition) or not. If they are steeper, this would indicate that there is "cross-habituation" within the set of manipulations of S6. Individual pairwise comparisons show that the habituation slopes are steeper for S6, S6R, S6RS and S6WN than for S1 $\left(Z_{c}<-1.72, p<0.042\right)$, for S6R and S6WN than for S7 $\left(Z_{c}<-1.78, p<0.038\right)$ and for S6R than for S5 $\left(Z_{c}=-1.83\right.$, $p=0.034)$, but not for the other combinations. If we treat each manipulation of S6 as a replicate of the same comparison, and 

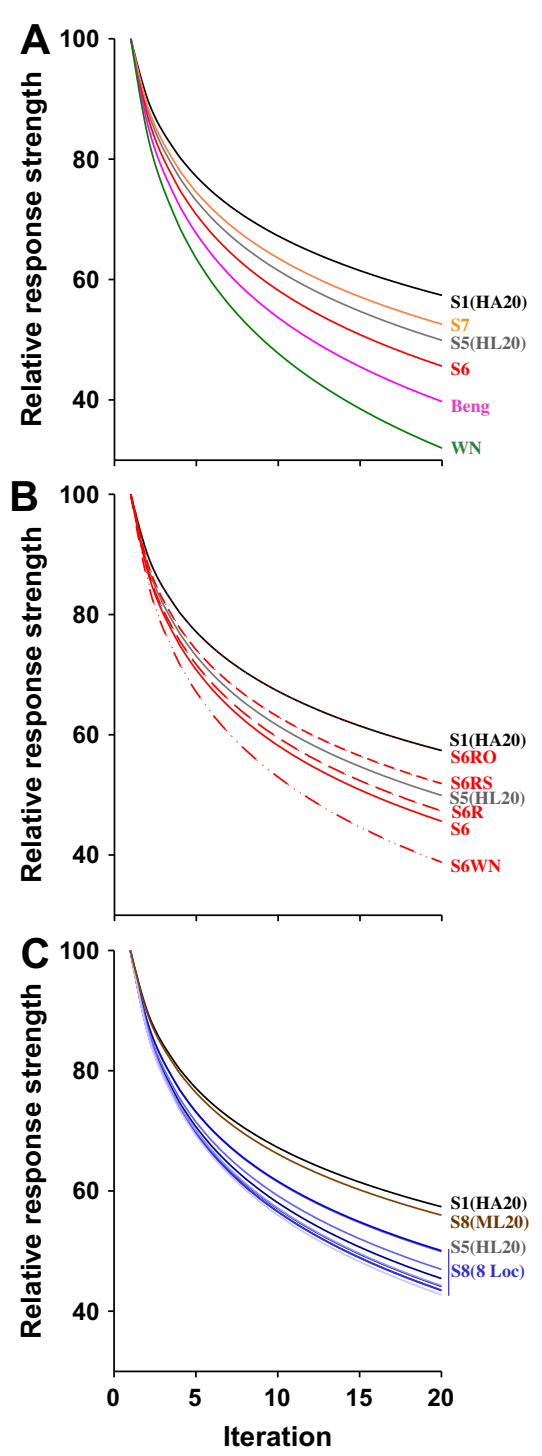

Fig. 5. Summary graphs of the habituation curves for the different conditions. The patterns are statistically supported by a Stouffer's test, which allows us to find overall significance even if many individual sites do not show significant differences between slopes. Significance in a Stouffer's test is achieved if many non-significant (and significant) sites all trend in the same direction. An example of a single site that follows the trend of the population is shown in Supplementary Fig. S8. (A) Comparison of the habituation curves from the first songs of the HabAcoustic (S1) and HabLocation (S5) conditions with 4 very different acoustic stimuli from the MixAcoustic condition. (B) Comparison of the habituation curves from the HabAcoustic (S1) and HabLocation (S5) conditions with 5 similar acoustic stimuli modeled on song 6 from the MixAcoustic condition. (C) Comparison of the habituation curves of the 8 different locations from the MixLocation condition (S8) to the curves from the HabAcoustic (S1) and HabLocation (S5) conditions. Different shades of blue indicate different locations from which the same song is being played. The brown line represents the habituation to the first 20 iterations of the song from the MixLocation condition (S8), regardless of location. Abbreviations as in Fig. 4, except HA20, habituation slope to the first 20 iterations of the first song of the HabAcoustic condition; HL20, habituation slope to the first 20 iterations of the first song of the HabLocation condition; ML20, habituation slope to the first 20 iterations of the song of the MixLocation condition, regardless of location.

combine the $p$-values of the 5 comparisons using Stouffer's method, we find that the 5 manipulations together have a significantly steeper slope than S1, S5 and S7 $\left(Z_{c}<-2.31, p<0.011\right.$ with S6WN; $Z_{c}<-1.90, p<0.03$ without S6WN). These overall findings indicate that the auditory responses habituate to songs in a similar way, whether they are played intermixed with other stimuli or not. The responses to the different versions of S6 (including the original version) habituate at a slightly higher rate than responses to the other zebra finch songs, probably because there are more renditions of versions of S6, and therefore they are treated as the first, fifth, ninth, etc. rendition of the same song, and therefore have a steeper habituation slope. That is, there appears to be cross-habituation to different versions of the same song (Figs. 5B and S8B).

In terms of other categories, we compared the habituation slopes of the zebra finch songs to those of the non-zebra-finch stimuli. We found that habituation slopes of responses to Bengalese finch song were significantly steeper than those to S1 and S7 $\left(Z_{c}<-2.370, p=0.009\right)$, but not than those to S5 or S6 $\left(Z_{c}>-1.065, p>0.13\right.$; Figs. 5A and S8A). Habituation slopes for responses to white noise were significantly steeper than those to $S 1$, S5 and S7 $\left(Z_{c}<-2.530, p<0.006\right)$, but not significantly steeper than those to S6 $\left(Z_{c}=-1.293, p=0.098\right.$; Figs. 5A and S8A). These findings indicates that the auditory system treats non-zebra-finch sounds differently from zebra finch sounds, reducing its response to them more quickly than to conspecific songs, but that it still treats the sounds independently of each other as it does with zebra finch songs.

\subsection{Habituation to a song played from 8 inter-mixed locations ignores the changes in location}

In an analogous experiment to the one in Section 3.3, to investigate whether the auditory system treats the same sound stimulus played from different locations as different auditory objects, we played the same song (S8) pseudo-randomly from 8 different locations (MixLocation condition; see Section 2.4). When we first looked at the habituation slopes, regardless of the location from which it was played, $77.3 \%$ (34/44) of sites habituated significantly to the first 50 renditions of S8 (Figs. 3E, 5C, S6 and S8C), which is similar to the proportion habituating to $\mathrm{S} 1$ in the HabAcoustic and S5 in the HabLocation conditions $\left(\chi^{2}(1)<0.27, p>0.604\right)$. The slopes based on the first 20 iterations were not significantly different from the slopes based on the first 20 iterations of S6 and S7 from the MixAcoustic, S5 from the HabLocation and S1 from the HabAcoustic conditions $\left(Z_{c}>-0.71, p>0.241\right.$; Figs. $5 C$ and $\mathrm{S} 8 \mathrm{C})$. These results suggest that the auditory system treats the repetition of the song as a single repeated event, independent of the continuous change in location. If this is true, then, because of cross-habituation, the habituation slopes for the 8 locations taken separately should each be significantly steeper than the habituation slopes calculated across all locations together, as well as than the habituation slopes to S1, S5 and S7 in the previous conditions.

We tested this idea by looking at the habituation slopes for the 8 locations separately. For 6 out of the 8 locations, the habituation slope was significantly steeper than for the first 20 renditions of S8 independent of location $\left(Z_{c}<-1.95, p<0.026\right.$; Figs. 3F, 5C, S7 and $\mathrm{S} 8 \mathrm{C}$ ). If we combine the 8 comparisons using Stouffer's method, the individual location slopes taken together are significantly steeper than the slope that ignores the location changes $\left(Z_{c}=-4.76\right.$, $p<0.0001)$. Similarly, the habituation slopes to the individual locations were significantly steeper than those to S7 from the MixAcoustic, to S1 from the HabAcoustic and to S5 from the HabLocation treatments $\left(Z_{c}<-4.47, p<0.0001\right.$; Figs. 5C and S8C). The habituation slopes for the 8 different locations taken together were also significantly steeper than the habituation slopes for the 4 variants of S6 from the MixAcoustic condition taken together $\left(Z_{c}=-3.51, p=0.0002\right)$. However, the habituation slopes for the 8 different locations were still shallower than the habituation slopes to the Bengalese finch song $\left(Z_{c}=-3.48, p=0.0002\right)$ and to the white noise stimulus $\left(Z_{c}=-5.05, p<0.0001\right)$. Taken together, these findings demonstrate that continuous changes in locations result in cross-habituation to the individual locations. 
A

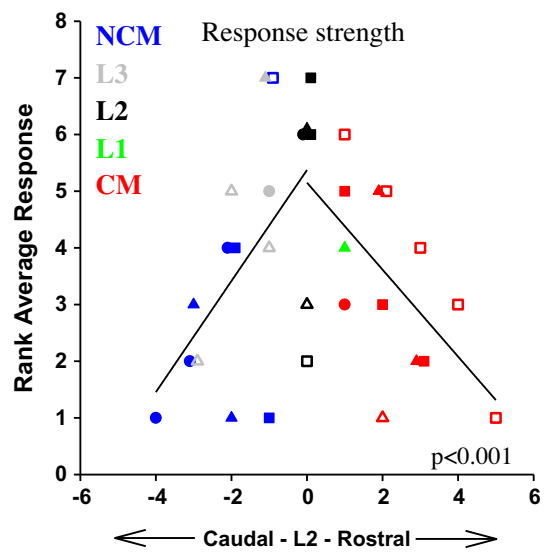

B

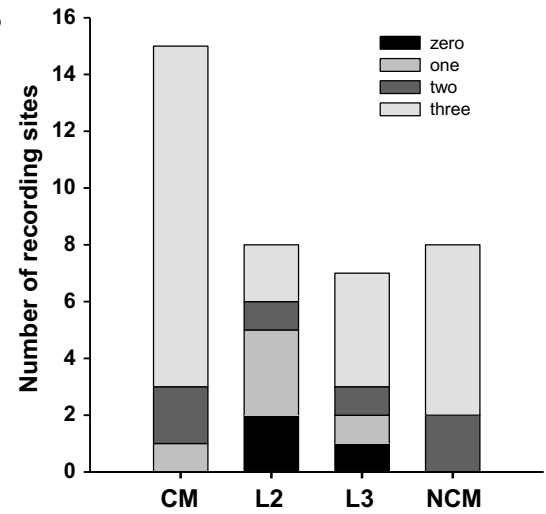

C

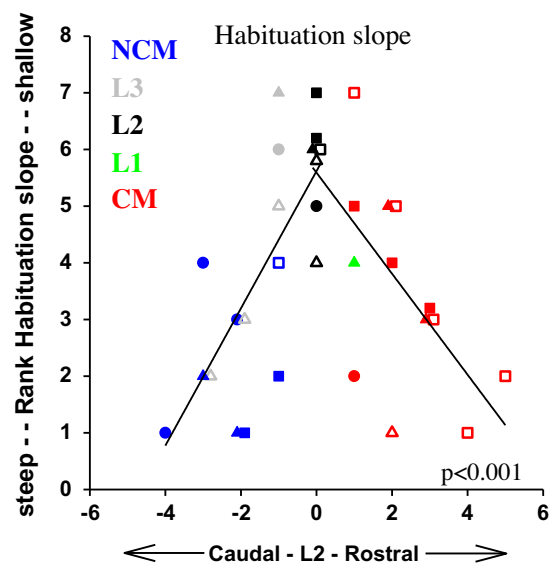

Fig. 6. Auditory response features and anatomical location. (A) Response strength to the first song in the HabAcoustic condition for all the sites in the 5 birds (symbols as in Fig. 2) included in that condition, ranked from lowest (1) to highest (7) for each individual bird ( $y$-axis) versus position within or relative to L2 ( $x$-axis). These ranks correlate negatively among the caudal and among the rostral locations relative to L2 (regression lines), and this is the case for all 4 songs in the HabAcoustic condition, as well as the songs in the other conditions (across 9 correlations, Spearman's Rho $<-0.547, p \leqslant 0.001(n=33-40))$. Overlapping points in the graph have been shifted slightly for clarity. Note that two birds have two recording sites each in L2. (B) Number of sites in each brain area (across all individuals) that habituated to the 50 iterations of S1, S5 and/or S8. The number of times we observed habituation in each site is filled in different shades of gray. Some sites habituated to all three songs, some only in two, one or in none. Note that the majority of sites in CM and NCM always habituated. (C) Similar analysis to A, but for the habituation slopes of all recording sites within an individual, ranked in such a way that more negative slopes (strongly habituating) receive low ranks, and less negative (or even positive) slopes get high ranks. This pattern was significant for the HabAcoustic condition shown and in all other conditions too (across 9 correlations, Spearman's Rho $<-0.393, p \leqslant 0.012(n=33-40))$. Colors as in Fig. 4A, indicating different brain areas. Symbols indicate different birds, as in Fig. 2.
3.5. Auditory responses are weaker but habituation slopes steeper downstream from Field $\mathrm{L} 2$

In addition to analyzing the data to test our hypothesis, we also investigated whether there were differences in the responsivity, habituation, and dishabituation among brain areas. For responsivity, the multiunit response strength in the different recording sites was very consistent from song to song (pair-wise rank correlations among the different songs in the HabAcoustic condition: Spearman's Rho $>0.895, p<0.001(n=36)$ ). Because of differences among birds in the quality of the recordings, we ranked the response strength among the recording sites for each bird for the responses to S1, S5 and S8. We then averaged those ranks and used a Kruskal-Wallis test to compare the mean ranks among the different brain areas. We found that the responses in L2 and L3 were consistently higher than those in CM and NCM $(\mathrm{K}-\mathrm{W}(3)=10.52$, $p=0.015)$. We also used the distance of a recording site from L2 along the electrode array as a proxy for anatomical location. This is roughly equivalent to distance downstream along the auditory pathway (Smith et al., 2006; Vates et al., 1996). We found that in each playback of a song the relative responses strengths were weaker the further downstream (anterior or posterior) the brain region from L2 (Fig. 6A).

For habituation, the habituation slopes among brain regions to repeated playback of stimuli were also consistent across songs (pair-wise rank correlations among S1-S4 for significantly habituating sites only: Spearman's Rho $>0.584, p<0.002(n=20-25)$ ). Across the three conditions for which we can measure habituation over the first 50 renditions of the first song (S1, S5 and S8), we found that sites in $\mathrm{CM}$ were more likely to show habituation to all three songs than sites in L2 (the only two brain areas for which we have recording sites in all birds; $\chi^{2}(1)=4.52, p=0.034$; Fig. $6 \mathrm{~B}$ ). A similar rank analysis to that used with the response strengths showed that the habituation slopes were also steeper in NCM and $\mathrm{CM}$ than in $\mathrm{L} 2$ and $\mathrm{L} 3(\mathrm{~K}-\mathrm{W}(3)=12.24, p=0.007)$. Accordingly, the slopes also were steeper the further downstream the recording site was from L2 (Fig. 6C).

For dishabituation, we found no evidence that dishabituating sites (either to a different song or to a change in location) were significantly associated with either a particular brain area $\left(\chi^{2}(3)<2.28, p>0.517\right)$ or with a given distance from L2 (MannWhitney $U>150, p>0.10$ ). We also did not find evidence to suggest that recording sites that dishabituate to a change in location were different in their distance from L2, compared to recording sites that dishabituate to a change in acoustic identity (MannWhitney $U=164, p=0.34$ ). These findings indicate that although response strength and habituation rates differ according to brain region, the differential mechanism of dishabituation to acoustic identity and location does not appear to differ according to brain region.

\section{Discussion}

Here we investigated habituation and dishabituation in the auditory system of awake, behaving birds. The main finding is that both a sudden change in acoustic properties and in spatial location lead to dishabituation of the auditory response. However, a continuous change among different acoustic stimuli leads to similar habituation to each stimulus separately, while a continuous change among different spatial locations does not.

\subsection{Potential explanations for the observed patterns of habituation and dishabituation}

Our finding that dishabituation happens both when the animal is suddenly exposed to a new acoustic stimulus and when the same 
acoustic stimulus is suddenly presented from a new location, is consistent with the IEG findings (Kruse et al., 2004). These authors interpreted their findings to indicate that the auditory pathway could form a memory that incorporated both acoustic identity and spatial location of a stimulus. However, we also found that responses to the same song played interleaved from 8 different locations habituate as if there were no changes in location at all. This rules out the idea that each song-location combination is treated as a separate auditory object.

We suggest that two different mechanisms may be responsible for the observed findings, possibly at the same time. The first process is the establishment of a neural code for acoustic stimuli in the auditory system. In locust antennal lobes, the precision with which a particular odor is encoded improves with repeated stimulation, which is accompanied by a decrease in the overall response to these stimuli (Stopfer \& Laurent, 1999). This is akin to the established interpretation of habituation in the songbird auditory system, that it represents the formation of memories (and hence fine-tuning of the response) for different acoustic stimuli (Chew et al., 1995; Chew et al., 1996). This explanation is also consistent with three other observations from our study. First, in the HabLocation condition, the overall response strength to the continued presentation of the same song steadily decreased across the three phases of the session, indicating continued habituation to the same song. Second, in the MixAcoustic condition, there appeared to be "cross-habituation" for the stimuli which were different manipulations of the same zebra finch song, which had very similar acoustic properties to each other. Finally, the response to each zebra finch song habituated separately, whether they were played interleaved with other stimuli or not. This process can also account for the dishabituation upon a change to a new acoustic stimulus, as a new memory for that stimulus needs to be laid down. Given that our recordings are multi-unit, it is likely that (at least in the higher-order brain areas) this dishabituation also represents an activation of a different subset of neurons in the small population of neurons we recorded from. Nevertheless, using measures of IEG protein and mRNA induced at different times, it has been shown that the same neuron that shows a habituated IEG response can respond to a subsequent novel auditory stimulus (Pinaud et al., 2008), and the analysis of single-unit responses shows a similar result (Chew et al., 1995). Therefore, the different subsets of neurons encoding different stimuli are expected to at least overlap. This hypothesis, however, does not account for dishabituation to a change in spatial location, given our findings that this cannot be interpreted as an integrative auditory object containing both stimulus identity and location information.

Our second hypothesis is that repeated presentation of the same stimulus leads to a habituation in the response to this stimulus as the stimulus becomes expected, and therefore less interesting for the auditory system and for the bird as a whole. In other words, each new rendition of the stimulus adds less and less new ethologically-relevant information to the bird. This hypothesis that response strength has something to do with the information gained being ethologically relevant is also supported by the fact that the response to white noise and to Bengalese finch noise habituated much more rapidly than the responses to zebra finch song. If habituation represents a decrease in ethologically-relevant information gain from a particular acoustic stimulus, then dishabituation must represent an increase in potential ethologically-relevant information gain. Like the first hypothesis, this mechanism can explain the response to the introduction of a novel acoustic stimulus, especially if it is an ethologically relevant stimulus. However, it also explains some other findings which are not so easily explained by the first hypothesis. First, the sudden onset of a song stimulus after a long period of silence is much unexpected (and hence adds extra information to be gained), and indeed, the response strength to the first song in the HabAcoustic condition was consistently higher than the response strengths to all the consequent songs, even though habituation occurred to all songs. Second, this hypothesis explains why dishabituation can also be induced by an unexpected non-acoustic change to an existing stimulus. We showed that an unexpected change in location of an acoustic stimulus induced dishabituation in a large proportion of recording sites, while a second change induced dishabituation in a significantly smaller proportion. We interpret this as a second change in location not being nearly as unexpected as the first one, and hence adding less new information. Expanding this interpretation to the MixLocation condition, it is then logical that there was no separate habituation for each location from which the song was played. After all, the expectation that the song was stationary was never built up in the first place. Instead, the song was mobile from the first iterations, and hence its continued mobility did not add any new information after the start of the session. The same interpretation is also consistent with published data on IEG expression. A novel pairing of foot-shock with a familiar song increases egr-1 expression in the canary auditory system (Jarvis et al., 1995). Similarly, light stimuli can reinstate the EGR-1 response to a habituated song when lights are then synchronized with the auditory stimulus (Kruse et al., 2004). All these cases could be interpreted as novel events, increasing the potential ethologically-relevant information that can be gained from the acoustic stimulus at that point in time, but only if they are clearly associated with the auditory stimulus.

Because our recordings are multi-unit, it is a legitimate question to wonder whether the dishabituation we observed with a sudden change of location represents a switch to a different subset of neurons in the ensembles we record from, or an increase in activity in the same neurons. We believe that dishabituation under hypothesis 2 might represent an increase in the activity of the same neurons, because the stimulus is acoustically identical, and would therefore be expected to be encoded by the same auditory network. The little that is known about spatial coding in the auditory forebrain suggests that neurons are sensitive to the egocentrically encoded location of the sound, and that neurons with similar spatial sensitivity are organized in clusters (Cohen \& Knudsen, 1998). To our knowledge, nothing is known about the encoding of allocentric spatial location in the auditory forebrain of birds, and there is still no clarity in mammals either (Altmann, Getzmann, \& Lewald, 2012; Schechtman, Shrem, \& Deouell, 2012).

It is possible that the observed habituation and dishabituation in the auditory systems are a combination of both mechanisms, as either by itself cannot explain all our findings. If both mechanisms act simultaneously, then one could do so by changing subpopulations of neurons involved in the auditory code and the other by modulating the firing activity of the same neurons.

The possibility that non-acoustic features can modulate auditory response strength if they indicate a potential increase in ethologically-relevant information (hypothesis 2) indicates a process very similar to attention at work. Indeed, Kruse et al. (2004) suggest that the habituation of the response to a repeated stimulus may be related to waning attention to that stimulus, and "dishabituation" to an increase in attention paid. Knowing that response strength is modulated by the potential ethological relevance of the stimulus, possibly mediated by an attention-like process, leads to the question of how this modulation is accomplished on a mechanistic level. Kruse et al. (2004) suggest a model for the enhancement of IEG expression in the auditory system, which can easily be extended to enhancement of electrophysiological activity as well. Under this model, different brain systems detect surprising events in different modalities. This detection in turn activates a brainstem arousal system that influences neural activity throughout the brain, and will enhance activity wherever it is simultaneously induced by 
another stimulus, potentially in another modality. In the case of the lights paired with the songs (Kruse et al., 2004), the novel lights would activate the arousal system. If song was played simultaneously, this song would then have induced a stronger response than it would have otherwise. This increased arousal response may then in turn associate the two stimuli with each other. If this model is true, in our experiment, a change in song location might have been detected by a brain system generally interested in allocentric spatial information (e.g. the hippocampus), which then affected the arousal system. This same brain system might not have detected any surprising events in the MixLocation condition, as none of the locations were unexpected (since no expectation was set up to begin with), and hence the habituation that took place was as would be expected from the same song being repeated many times.

\subsection{Anatomical location affects strength of auditory response and rate of habituation}

We also produced some new findings on anatomical location and auditory response strength. First, we found that the auditory telencephalon of zebra finches responds preferentially to stimulation from the contralateral auditory hemifield. This is consistent with: (1) Findings in L2 of head-fixed non-songbirds hearing sounds (owls (Cohen \& Knudsen, 1998), chicks (Scheich, 1983), and doves (Biederman-Thorson, 1970)); (2) the ascending auditory pathway in zebra finches which shows a predominantly contralateral projection from the brain stem nuclei to the midbrain and ipsilateral from there to the forebrain (Wild, Krützfeldt, \& Kubke, 2010); (3) reduction in contralateral hearing-induced EGR-1 expression response after plugging one ear during song playback (Feenders et al., 2008); and (4) the improved ability to distinguish a target from a masking stimulus if the target is presented on the contralateral side and the masker on the ipsilateral side compared to the opposite scenario (Maddox, Billimoria, Perrone, Shinn-Cunningham, \& Sen, 2012). It is also similar to the mammalian auditory system (Glendenning, Baker, Hutson, \& Masterton, 1992).

Second, we found that sites in L2 habituated to repeated stimulation, although the rate of habituation is lower (i.e. the slope is shallower) than that of higher-order sites. Indeed, the further away the recording sites were from $\mathrm{L} 2$, the more their response strength to our stimuli decreased and the steepness of their habituation slopes increased. This trend relative to $\mathrm{L} 2$ seems to run through a range of brain areas, and is evident even within NCM and within CM. Although it is well established that L2 is electrophysiologically responsive to auditory stimulation (e.g. Gehr, Capsius, Grabner, Gahr, \& Leppelsack, 1999), we are not aware of any reports of habituation to repeated song stimulation in L2 (Chew et al., 1995; Chew et al., 1996; Stripling et al., 1997). In fact, it has been often assumed not to occur since the IEGs studied were not induced there. In studies looking at egr-1 expression, L2 cannot be studied from the point of view of habituation, as it does not expresses much egr-1 to begin with, compared to the surrounding brain areas (Mello \& Clayton, 1994). It does, however, express another IEG, called dusp1, which is induced in L2, but not higher order auditory neurons, upon song presentation (Horita et al., 2010); possible habituation of dusp1 expression has not yet been studied.

One possible explanation for a relationship between a change in response strength and a change in habituation slope with distance from L2 could be related to the proportion of neurons in the recorded local population that respond to the stimulus. Because the response of individual neurons is more selective in higherorder auditory areas, fewer neurons there might respond to any given stimulus, while nearer L2, the neurons are less selective, and therefore more of them might respond to a given stimulus (Theunissen et al., 2004). This would lead to a weaker multi-unit response the further away from $\mathrm{L} 2$. Conversely to the response strength, the pattern in the differences in the habituation slopes could be explained if a smaller proportion of the responsive neurons near L2 habituated to the stimulus, while a larger proportion of the responsive neurons farther away from L2 habituated to the stimulus. This would then result in shallower multi-unit habituation slopes near L2. Indeed, it is even possible that L2 neurons themselves do not actually habituate, but that the shallow habituation we have detected in some of our L2 recording sites is due to habituating neurons in adjacent regions to L2, which were close enough to the electrode to be picked up. Regardless of the explanation, the fact remains that more of the responsive neurons seem to habituate to repeated stimulation with the same song in higher than in lower-order auditory areas.

Finally, we showed for the first time that the caudal striatum in birds, a region that shows a hearing-induced IEG response (Jarvis et al., 2000), shows auditory neurophysiological responsiveness and that this response can habituate to repeated stimulation. Because we only had very few electrodes in this area, we cannot say much more about it at this point in time.

\section{Conclusion}

Habituation to repeated exposure to the same stimulus, and reinstatement of a stronger response after a change to a different stimulus happens in awake and behaving animals, just like it does in anaesthetized and restrained animals. This suggests strongly that the habituation is an ethologically relevant phenomenon, and not an artifact of the fact that the bird is not actively interacting with its world. Our results, in combination with the literature, suggest that dishabituation of auditory responses may represent a combination of two processes: one involved in encoding memories of auditory stimuli, and the second involved in the detection of salient stimuli in the environment. This salience may be signaled both by their acoustic properties (e.g. the appearance of a new bird in the environment) and non-acoustic properties (e.g. an unexpected change in location of a heretofore stationary stimulus). As such, the auditory response strength to a given stimulus may be related to how much attention a bird pays to a stimulus. It will be interesting to test this hypothesis in the future in a study where attention can be measured behaviorally, while simultaneously recording from the auditory system.

\section{Acknowledgments}

We thank Deepa Sambandan for assistance in data collection. During the experimental work described in this paper, TVS was partially supported by an institutional NRSA post-doctoral training grant held in the Department of Neurobiology at Duke University Medical Center. The work was funded by a David and Lucille Packard Foundation Award, and a NSF \& NIH CRCNS R01-DC007996 from NIDCD to EDJ.

\section{Appendix A. Supplementary material}

Supplementary data associated with this article can be found, in the online version, at http://dx.doi.org/10.1016/j.nlm.2013.08.010.

\section{References}

Altmann, C. F., Getzmann, S., \& Lewald, J. (2012). Allocentric or craniocentric representation of acoustic space: An electrotomography study using mismatch negativity. PLoS One, 7(7), e41872. http://dx.doi.org/10.1371/journal.pone.

Barbati, G., Porcaro, C., Zappasodi, F., Rossini, P. M., \& Tecchio, F. (2004). Optimization of an independent component analysis approach for artifact identification and removal in magnetoencephalographic signals. Clinical Neurophysiology, 115(5), 1220-1232. 
Biederman-Thorson, M. (1970). Auditory evoked responses in the cerebrum (field L) and ovoid nucleus of the ring dove. Brain Research, 24(2), 235-245.

Bornstein, M. H. (1985). Habituation of attention as a measure of visual information processing in human infants: Summary, systematization, and synthesis. In G. Gottlieb \& N. A. Krasnegor (Eds.), Measurement of audition and vision in the first year of postnatal life: A methodological overview (pp. 253-300). Norwood, NJ: Ablex.

Brown, G. D., Yamada, S., \& Sejnowski, T. J. (2001). Independent component analysis at the neural cocktail party. Trends in Neurosciences, 24(1), 54-63.

Chew, S. J., Mello, C., Nottebohm, F., Jarvis, E., \& Vicario, D. S. (1995). Decrements in auditory responses to a repeated conspecific song are long-lasting and require two periods of protein synthesis in the songbird forebrain. Proceedings of the National Academy of Sciences of the United States of America, 92(8), 3406-3410.

Chew, S. J., Vicario, D. S., \& Nottebohm, F. (1996). A large-capacity memory system that recognizes the calls and songs of individual birds. Proceedings of the National Academy of Sciences of the United States of America, 93(5), 1950-1955.

Cohen, Y. E., \& Knudsen, E. I. (1998). Representation of binaural spatial cues in field L of the barn owl forebrain. Journal of Neurophysiology, 79(2), 879-890.

Delorme, A., \& Makeig, S. (2004). EEGLAB: An open source toolbox for analysis of single-trial EEG dynamics including independent component analysis. Journal of Neuroscience Methods, 134(1), 9-21.

DiCarlo, J. J., Lane, J. W., Hsiao, S. S., \& Johnson, K. O. (1996). Marking microelectrode penetrations with fluorescent dyes. Journal of Neuroscience Methods, 64(1), 75-81.

Feenders, G., Liedvogel, M., Rivas, M., Zapka, M., Horita, H., Hara, E., et al. (2008). Molecular mapping of movement-associated areas in the avian brain: A motor theory for vocal learning origin. PLoS One, 3(3), e1768. http://dx.doi.org/ 10.1371 /journal.pone.0001768.

Fisher, N. I. (1993). Statistical analysis of circular data. Cambridge, UK: Cambridge University Press.

Gehr, D. D., Capsius, B., Grabner, P., Gahr, M., \& Leppelsack, H. J. (1999). Functional organisation of the field-L-complex of adult male zebra finches. NeuroReport, 10(2), 375-380.

Gill, P., Woolley, S. M. N., Fremouw, T., \& Theunissen, F. E. (2008). What's that sound? Auditory area CLM encodes stimulus surprise, not intensity or intensity changes. Journal of Neurophysiology, 99(6), 2809-2820. http://dx.doi.org/ $10.1152 /$ jn.01270.2007.

Glendenning, K. K., Baker, B. N., Hutson, K. A., \& Masterton, R. B. (1992). Acoustic chiasm V: Inhibition and excitation in the ipsilateral and contralateral projections of LSO. Journal of Comparative Neurology, 319(1), 100-122. http:// dx.doi.org/10.1002/cne.903190110.

Horita, H., Wada, K., Rivas, M. V., Hara, E., \& Jarvis, E. D. (2010). The dusp1 immediate early gene is regulated by natural stimuli predominantly in sensory input neurons. Journal of Comparative Neurology, 518(14), 2873-2901. http:// dx.doi.org/10.1002/cne.22370.

Iriarte, J., Urrestarazu, E., Valencia, M., Alegre, M., Malanda, A., Viteri, C., et al. (2003). Independent component analysis as a tool to eliminate artifacts in EEG: A quantitative study. Journal of Clinical Neurophysiology, 20(4), 249-257.

Jarvis, E. D. (2004). Learned birdsong and the neurobiology of human language. Annals of the New York Academy of Sciences, 1016, 749-777.

Jarvis, E. D., Güntürkün, O., Bruce, L., Csillag, A., Karten, H., Kuenzel, W., et al. (2005). Avian brains and a new understanding of vertebrate brain evolution. Nature Reviews Neuroscience, 6(2), 151-159.

Jarvis, E. D., Mello, C. V., \& Nottebohm, F. (1995). Associative learning and stimulus novelty influence the song-induced expression of an immediate early gene in the canary forebrain. Learning and Memory, 2(2), 62-80.

Jarvis, E. D., \& Nottebohm, F. (1997). Motor-driven gene expression. Proceedings of the National Academy of Sciences of the United States of America, 94(8), 4097-4102.

Jarvis, E. D., Ribeiro, S., da Silva, M. L., Ventura, D., Vielliard, J., \& Mello, C. V. (2000). Behaviourally driven gene expression reveals song nuclei in hummingbird brain. Nature, 406(6796), 628-632.

Jarvis, E. D., Smith, V. A., Wada, K., Rivas, M. V., McElroy, M., Smulders, T. V., et al. (2002). A framework for integrating the songbird brain. Journal of Comparative Physiology A - Neuroethology Sensory Neural and Behavioral Physiology, 188(112), 961-980. http://dx.doi.org/10.1007/s00359-002-0358-y.

Joyce, C. A., Gorodnitsky, I. F., \& Kutas, M. (2004). Automatic removal of eye movement and blink artifacts from EEG data using blind component separation. Psychophysiology, 41(2), 313-325.

Jung, T. P., Makeig, S., Westerfield, M., Townsend, J., Courchesne, E., \& Sejnowski, T. J. (2000). Removal of eye activity artifacts from visual event-related potentials in normal and clinical subjects. Clinical Neurophysiology, 111(10), 1745-1758.

Kruse, A. A., Stripling, R., \& Clayton, D. F. (2004). Context-specific habituation of the zenk gene response to song in adult zebra finches. Neurobiology of Learning and Memory, 82(2), 99-108.

Lu, K., \& Vicario, D. S. (2011). Toward a neurobiology of auditory object perception: What can we learn from the songbird forebrain? Current Zoology, 57(6), 671-683.

Maddox, R. K., Billimoria, C. P., Perrone, B. P., Shinn-Cunningham, B. G., \& Sen, K. (2012). Competing sound sources reveal spatial effects in cortical processing.
PLoS Biology, 10(5). ARTN e1001319, doi:http://dx.doi.org/10.1371/ journal.pbio.1001319.

Makeig, S., Bell, A. J., Jung, T. P., \& Sejnowski, T. J. (1996). Independent componen analysis of electroencephalographic data. In D. Touretzky, M. Mozer, \& M. E. Hasselmo (Eds.), Advances in neural information processing systems (pp. 145-151). Cambridge, MA: MIT Press.

Makeig, S., et al. (2000). ICA toolbox for psychophysiological research (version 3.4). <www.cnl.salk.edu/ ica.html>.

Mello, C. V., \& Clayton, D. F. (1994). Song-induced ZENK gene expression in auditory pathways of songbird brain and its relation to the song control system. Journal of Neuroscience, 14(11), 6652-6666.

Mello, C. V., Nottebohm, F., \& Clayton, D. (1995). Repeated exposure to one song leads to a rapid and persistent decline in an immediate early gene's response to that song in zebra finch telencephalon. Journal of Neuroscience, 15(10), 6919-6925.

Mello, C. V., Pinaud, R., \& Ribeiro, S. (1998). Noradrenergic system of the zebra finch brain - Immunocytochemical study of dopamine-beta-hydroxylase. Journal of Comparative Neurology, 400(2), 207-228.

Moorman, S., Mello, C. V., \& Bolhuis, J. J. (2011). From songs to synapses: Molecular mechanisms of birdsong memory. BioEssays, 33(5), 377-385. http://dx.doi.org/ 10.1002/bies.201000150.

Naselaris, T., Merchant, H., Amirikian, B., \& Georgopoulos, A. P. (2005). Spatial reconstruction of trajectories of an array of recording microelectrodes. Journal of Neurophysiology, 93(4), 2318-2330.

Park, K. H. J., \& Clayton, D. F. (2002). Influence of restraint and acute isolation on the selectivity of the adult zebra finch zenk gene response to acoustic stimuli. Behavioural Brain Research, 136(1), 185-191.

Pinaud, R., Mello, C. V., Velho, T. A., Wynne, R. D., \& Tremere, L. A. (2008). Detection of two mRNA species at single-cell resolution by double-fluorescence in situ hybridization. Nature Protocols, 3(8), 1370-1379. http://dx.doi.org/10.1038/ nprot.2008.115.

Ribeiro, S., \& Mello, C. V. (2000). Gene expression and synaptic plasticity in the auditory forebrain of songbirds. Learning $\mathcal{E}$ Memory, 7(5), 235-243.

Rosen, M. J., \& Mooney, R. (2000). Intrinsic and extrinsic contributions to auditory selectivity in a song nucleus critical for vocal plasticity. Journal of Neuroscience, 20(14), 5437-5448.

Rosen, M. J., \& Mooney, R. (2003). Inhibitory and excitatory mechanisms underlying auditory responses to learned vocalizations in the songbird nucleus HVC. Neuron, 39(1), 177-194.

Schechtman, E., Shrem, T., \& Deouell, L. Y. (2012). Spatial localization of auditory stimuli in human auditory cortex is based on both headindependent and head-centered coordinate systems. Journal of Neuroscience, 32(39), 13501-13509.

Scheich, H. (1983). Two columnar systems in the auditory neostriatum of the chick: Evidence from 2-deoxyglucose. Experimental Brain Research, 51(2), 199-205.

Smith, V. A., Yu, J., Smulders, T. V., Hartemink, A. J., \& Jarvis, E. D. (2006). Computational inference of neural information flow networks. PLoS Computational Biology, 2(11), e161.

Stopfer, M., \& Laurent, G. (1999). Short-term memory in olfactory network dynamics. Nature, 402(6762), 664-668. http://dx.doi.org/10.1038/45244.

Stripling, R., Volman, S. F., \& Clayton, D. F. (1997). Response modulation in the zebra finch neostriatum: Relationship to nuclear gene regulation. Journal of Neuroscience, 17(10), 3883-3893.

Theunissen, F. E., Amin, N., Shaevitz, S. S., Woolley, S. M. N., Fremouw, T., \& Hauber, M. E. (2004). Song selectivity in the song system and in the auditory forebrain. Annals of the New York Academy of Sciences, 1016(1), 222-245.

Tran, Y., Craig, A., Boord, P., \& Craig, D. (2004). Using independent component analysis to remove artifact from electroencephalographic measured during stuttered speech. Medical E' Biological Engineering E' Computing, 42(5), 627-633.

Vates, G. E., Broome, B. M., Mello, C. V., \& Nottebohm, F. (1996). Auditory pathways of caudal telencephalon and their relation to the song system of adult male zebra finches (Taenopygia guttata). Journal of Comparative Neurology, 366(4) 613-642.

Wang, Y., Brzozowska-Prechtl, A., \& Karten, H. J. (2010). Laminar and columnar auditory cortex in avian brain. Proceedings of the National Academy of Sciences of the United States of America. http://dx.doi.org/10.1073/ pnas.1006645107.

Whitlock, M. C. (2005). Combining probability from independent tests: The weighted Z-method is superior to Fisher's approach. Journal of Evolutionary Biology, 18(5), 1368-1373. http://dx.doi.org/10.1111/j.1420-9101.2005. 00917.x.

Wild, J. M., Krützfeldt, N. O. E., \& Kubke, M. F. (2010). Connections of the auditory brainstem in a songbird, Taeniopygia guttata. III. Projections of the superior olive and lateral lemniscal nuclei. Journal of Comparative Neurology, 518(11) 2149-2167. http://dx.doi.org/10.1002/cne.22325. 